\title{
Mapping the Gauss Coefficients to the Pole and the Models of Paleosecular Variation
}

\author{
Masaru KONO ${ }^{1}$ and Hidefumi TANAKA ${ }^{2}$ \\ ${ }^{1}$ Department of Earth and Planetary Physics, University of Tokyo, \\ Yayoi 2-11-16, Bunkyo-ku, Tokyo 113, Japan \\ 2 Department of Earth and Planetary Sciences, Tokyo Institute of Technology, \\ Ookayama 2-12-1, Meguro-ku, Tokyo 152, Japan
}

(Received January 12, 1994; Revised October 13, 1994; Accepted October 20, 1994)

\begin{abstract}
The present magnetic field is usually described by models such as the IGRF which are described by Gauss coefficients obtained by spherical harmonic analysis of the geomagnetic field. The use of Gauss coefficients in paleosecular variation (PSV) studies will therefore make it possible to compare field structures obtained through paleomagnetism with those observed directly. For making such comparisons, it is important to understand how the magnetic field maps into geomagnetic poles and what are their statistical properties, because geomagnetic poles are frequently used in PSV studies. We show how Gauss coefficients map into pole positions, and we develop statistical tools to treat the angular scatter of magnetic poles calculated from models based on Gauss coefficients. We use these methods to evaluate the models of Constable and Parker (1988) and McFadden et al. (1988) which are typical of PSV models using Gauss coefficients. Our results suggests the importance of the $(\ell=2, m=1)$ harmonic in the dynamo process of the earth.
\end{abstract}

\section{Introduction}

In the 1960s and 1970s, the study of paleosecular variation (PSV) progressed considerably through the work of the late Allan Cox, especially through his paper of 1970 . In that paper, Cox showed how magnetic field directions are mapped into poles or the poles into directions, and he demonstrated the statistical properties of the distribution of projected values (poles, directions) when the original data (directions, poles) follow a Fisher (1953) distribution. Because the main source of data used in PSV studies are lavas which record instantaneous directions of the magnetic field, the poles mapped from the field directions are what we usually call virtual geomagnetic poles (VGPs), which are field directions mapped into poles with the assumption of the geocentric dipole. Cox also developed methods for describing the separate contributions from the dipole and nondipole fields to the angular dispersion of either field directions or poles. It may be concluded that Models C and D of Cox (1970) can be considered as the typical of the classical PSV models.

In these classical PSV models, it was recognized that the present geomagnetic field is dominated by the dipole term. It was assumed, partly because of the problem of resolution and the paucity of data which prevented the measurement of PSV at different longitudes in a latitudinal band, and partly because of the belief that the fluctuations in the same latitudinal band should be averaged out in a short time $\left(\approx 10^{3}\right.$ years $)$, that the long term secular variation can be described by the latitude dependence of the angular standard deviation (ASD) of either directions or of VGPs. The angular variance (which is equivalent to the square of the ASD) of the VGPs is defined as

$$
S^{2}=\frac{1}{N-1} \sum_{i=1}^{N} \Delta_{i}^{2},
$$


where $\Delta_{i}$ denotes the angular distance of the $i$ th pole from the mean and $N$ is the number of observed VGPs. The denominator $N-1$ is replaced by $N$ if the mean pole position is known $a$ priori.

In spite of the marked difference of the nondipole field in the two hemispheres at present, it was also assumed that the north-south asymmetry is temporary, and that the time-averaged magnetic field must have symmetry about the equator. The ASD of the VGP can be divided into two parts of which one is caused by the dipole and the other by nondipole sources. As all the longitudinal variations were assumed to be averaged out over a sufficiently long interval, the objective of the classical PSV models was to explain the latitude dependence of the ASD. Thus the angular variance for a classical PSV model can be represented by a model

$$
S^{2}=S_{D}^{2}+F(\lambda) W_{N}^{2}
$$

where $S_{D}^{2}$ is the latitude-independent variance caused by the dipole fluctuations (dipole wobble), $W_{N}^{2}$ is a constant showing the contribution from the nondipole field, and $F(\lambda)$ is an even function of latitude $\lambda$. In some cases, $F(\lambda)$ was assumed to depend not only on latitude but on other parameters (e.g., dipole intensity in the case of Model D of Cox). The observed ASD increases monotonically with latitude (Fig. 1), and so a function $F(\lambda)$ which produced such a trend was sought.

Recently, new models of paleosecular variation have been proposed by Constable and Parker (1988) and by McFadden et al. (1988). They both utilize the Gauss coefficients in defining their models. Since expressions with Gauss coefficients are universally used in the analysis of the recent geomagnetic field, and since spherical harmonic analysis is a complete way to describe the potential field, these new models brought PSV studies based on paleomagnetic data much closer to the analyses applied to the present geomagnetic field.

Constable and Parker (1988) noted the behavior of the power of the present magnetic field

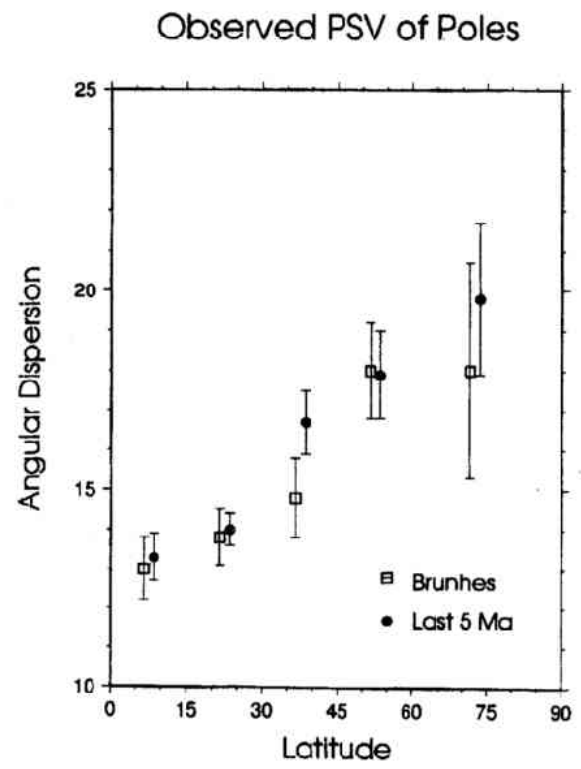

Fig. 1. Paleomagnetically observed angular dispersion of the VGPs in the Brunhes chron and in the last 5 million years from McElhinny and Merrill (1975). Values of the ASD are shown for latitude bands of $15^{\circ}$ intervals starting at the equator, except the last point which are for all the data between $60^{\circ}$ and $90^{\circ}$. 
in spherical harmonic degree $\ell$ at radius $r$ as defined by Lowes (1974)

$$
R_{\ell}(r)=\left(\frac{a}{r}\right)^{2(\ell+2)}(\ell+1) \sum_{m=0}^{\ell}\left\{\left(g_{\ell}^{m}\right)^{2}+\left(h_{\ell}^{m}\right)^{2}\right\},
$$

where $a$ is the mean radius of the earth and $g_{\ell}^{m}$ and $h_{\ell}^{m}$ are the Gauss coefficients of degree $\ell$ and order $m$. At the earth's surface $(r=a)$, the spectrum drops exponentially with a slope indicating a white spectrum slightly below the core's surface (Langel and Estes, 1982). Figure 2 is a diagram showing the relation between harmonic degree $\ell$ and the power $R_{\ell}$ for the magnetic fields of $1990,1890,1790$, and 1690 , based on the recent secular variation model of Bloxham and Jackson (1992). The exponential drop of power between degrees 2 and about 8 is apparent for all the models. The deviation from an exponential trend at higher degrees is brought about by the requirement of smoothness at the surface of the core, which was imposed in the inversion of data (Bloxham and Jackson, 1992), and is not important in the present discussion. From this observation, Constable and Parker (1988) assumed that, excluding the dipole terms, the Gauss coefficients of the same degree $\ell, g_{\ell}^{m}, h_{\ell}^{m}$, obey a normal distribution with zero mean and variance $\sigma_{\ell}^{2}$, where

$$
\sigma_{\ell}^{2}=\frac{(c / a)^{2 \ell} \alpha^{2}}{(\ell+1)(2 \ell+1)},
$$

where $c$ is the radius of the core and $\alpha$ is a constant having the dimension of magnetic field. This corresponds to the assumption that the power $R_{\ell}$ has an exactly flat spectrum at the surface of the core. By this choice, the rescaled Gauss coefficients

$$
\tilde{g}_{\ell}^{m}=v_{l} g_{\ell}^{m}, \quad \tilde{h}_{\ell}^{m}=v_{l} h_{\ell}^{m},
$$

where $v_{l}^{2}=(\ell+1)(2 \ell+1)(a / c)^{2 \ell}$, are independent samples of a single zero mean Gaussian process (which Constable and Parker called the giant Gaussian process). As the power of the dipole terms

\section{Geomagnetic Power Spectrum 1690-1990}

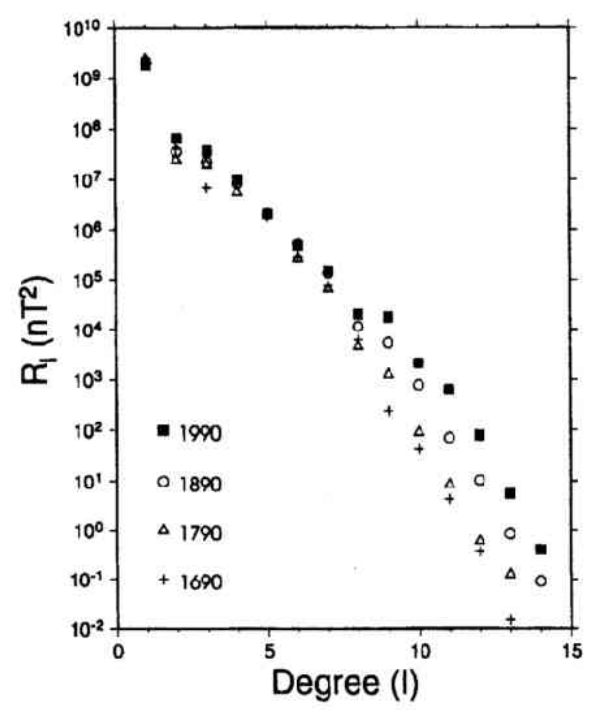

Fig. 2. The power of the geomagnetic field at 100-year intervals starting from 1990 back to 1690 based on the secular variation model of Bloxham and Jackson (1992). 
does not follow the trend shown by the terms between $\ell=2$ and $8, g_{1}^{0}, g_{1}^{1}, h_{1}^{1}$ were assumed to be random variables, but different from others. They had also to assume that although $g_{2}^{0}$ belongs to the giant Gaussian process, it has a nonzero mean to be consistent with the paleomagnetic data compiled by Lee (1983).

McFadden et al. (1988) noted that the N-S asymmetry in the present field is nearly removed if the equatorial dipole terms $g_{1}^{1}, h_{1}^{1}$ are rotated by $90^{\circ}$, which strongly suggests that the asymmetry is a temporary feature. Further, the ASDs of VGPs at present and in the last $5 \mathrm{Ma}$ measured with respect to the rotational axis agree well with each other. When they divided the Gauss coefficients into dipole family (harmonics for which $\ell+m=$ odd) and quadrupole family $(\ell+m=$ even), based on the separation of solutions in some dynamo models (Roberts and Stix, 1972), they found that the angular dispersion of the present magnetic field due to the dipole family is proportional to latitude, while that due to the quadrupole family is nearly constant and independent of latitude. Therefore, they concluded that the total variance can be approximated as

$$
S^{2}=A^{2} \lambda^{2}+B^{2},
$$

where the first and second terms on the right hand side indicate the contributions from dipole and quadrupole families, and $A$ and $B$ are constants at any given epoch but may change with time.

These new models attempt to explain PSV (observed by paleomagnetism) through models based on Gauss coefficients (the means of description for the present field). These models are very attractive in making it possible to compare the paleomagnetic field with the present and recently observed field. However, it is not clear how these two models compare with each other or with other PSV models. It should also be noted that Constable and Parker (1988) did not show how ASD changes with latitude in their model, in spite of the fact that it is generally the most frequently used relation in PSV studies. For such analysis, it is necessary to understand how a magnetic field, described by a combination of Gauss coefficients, maps to a VGP and what kind of statistical properties are expected for the ensemble of these poles. We will address this problem in this paper.

\section{Mapping the Gauss Coefficients to the Pole}

In this section, we will derive the formulas needed to construct the relation between the latitude and the angular standard deviation (ASD) of the virtual magnetic poles (VGPs) calculated when the model is given in terms of the Gauss coefficients.

\subsection{Field elements}

The potential of the geomagnetic field at a site with colatitude $\theta(=\pi / 2-\lambda$, where $\lambda$ is the latitude) and longitude $\phi$ is expressed in the standard form

$$
W=a \sum_{\ell=1}^{\infty} \sum_{m=0}^{\ell}\left(\frac{a}{r}\right)^{\ell+1}\left(g_{\ell}^{m} \cos m \phi+h_{\ell}^{m} \sin m \phi\right) P_{\ell}^{m}(\cos \theta),
$$

where $r$ is the radial coordinate, $a$ is the earth's mean radius, and $P_{\ell}^{m}$ is the Schmidt-normalized Legendre function

$$
\begin{aligned}
P_{\ell}^{0}(x) & =P_{\ell}(x)=\frac{1}{2^{\ell} \ell !} \frac{d^{\ell}}{d x^{\ell}}\left(x^{2}-1\right)^{\ell}, \quad m=0, \\
P_{\ell}^{m}(x) & =\sqrt{2 \frac{(\ell-m) !}{(\ell+m) !}}\left(1-x^{2}\right)^{m / 2} \frac{d^{m} P_{\ell}(x)}{d x^{m}}, \quad 0<m \leq \ell,
\end{aligned}
$$


which satisfies the orthogonality condition

$$
\iint P_{\ell}^{m}(\cos \theta)\left(\begin{array}{l}
\cos m \phi \\
\sin m \phi
\end{array}\right) P_{\ell^{\prime}}^{m^{\prime}}(\cos \theta)\left(\begin{array}{c}
\cos m^{\prime} \phi \\
\sin m^{\prime} \phi
\end{array}\right) \sin \theta d \theta d \phi=\frac{4 \pi}{2 \ell+1} \delta_{\ell \ell^{\prime}} \delta_{m m^{\prime}} .
$$

If we neglect the ellipticity of the earth, the downward-, northward-, and eastward-components of the magnetic field at the surface can be derived from the potential as

$$
\begin{aligned}
& Z=\left.\frac{\partial W}{\partial r}\right|_{r=a}=-\sum_{\ell} \sum_{m}(\ell+1)\left(g_{\ell}^{m} \cos m \phi+h_{\ell}^{m} \sin m \phi\right) P_{\ell}^{m}(\cos \theta), \\
& X=\left.\frac{\partial W}{r \partial \theta}\right|_{r=a}=\sum_{\ell} \sum_{m}\left(g_{\ell}^{m} \cos m \phi+h_{\ell}^{m} \sin m \phi\right) \frac{d P_{\ell}^{m}}{d \theta} \\
& Y=-\left.\frac{\partial W}{r \sin \theta \partial \phi}\right|_{r=a}=\sum_{\ell} \sum_{m}\left(g_{\ell}^{m} \sin m \phi-h_{\ell}^{m} \cos m \phi\right) \frac{m P_{\ell}^{m}}{\sin \theta}
\end{aligned}
$$

The inclination $(I)$ and declination $(D)$ of the magnetic field can be expressed in terms of the components of the magnetic field vector as

$$
\tan I=\frac{Z}{\sqrt{X^{2}+Y^{2}}}, \quad \tan D=\frac{Y}{X} .
$$

\subsection{Mapping the field direction to the pole: dipole case}

The first problem is to find out the way in which the field directions derived from the geomagnetic potential are mapped into VGP distributions. A field direction $(I, D)$ obtained at a site $(\theta, \phi)$ will be converted to a VGP with colatitude $\theta_{p}$ and longitude $\phi_{p}$ through the use of relations of spherical trigonometry

$$
\cos \theta_{p}=\cos \theta \cos \psi+\sin \theta \sin \psi \cos D, \quad \cos \left(\phi_{p}-\phi\right)=\frac{\cos \psi-\cos \theta \cos \theta_{p}}{\sin \theta \sin \theta_{p}},
$$

where $\psi$ is the angular distance from the site to the VGP given by the dipole formula $\tan I=$ $2 \cot \psi$. In the following we do not need the complete description of the pole given by the above formula. Instead, we use the relations for the angles $\psi$ and $D$,

$$
\cos \psi=\frac{Z / 2}{\sqrt{X^{2}+Y^{2}+(Z / 2)^{2}}}, \quad \sin \psi=\frac{\sqrt{X^{2}+Y^{2}}}{\sqrt{X^{2}+Y^{2}+(Z / 2)^{2}}}, \quad \cos D=\frac{X}{\sqrt{X^{2}+Y^{2}}} .
$$

With these relations, the colatitude $\theta_{p}$ of the VGP in the present coordinate system is written as

$$
\cos \theta_{p}=\cos \theta \cos \psi+\sin \theta \sin \psi \cos D=\frac{(Z / 2) \cos \theta+X \sin \theta}{\sqrt{X^{2}+Y^{2}+(Z / 2)^{2}}} .
$$

In the case that the field is composed of the dipole terms $(\ell=1)$ only, the field components are

$$
\begin{array}{rrr}
Z / 2 & = & -g_{1}^{0} \cos \theta-g_{1}^{1} \sin \theta \cos \phi-h_{1}^{1} \sin \theta \sin \phi, \\
X & = & -g_{1}^{0} \sin \theta+g_{1}^{1} \cos \theta \cos \phi+h_{1}^{1} \cos \theta \sin \phi, \\
Y & = & g_{1}^{1} \sin \phi-h_{1}^{1} \cos \phi .
\end{array}
$$

For this case, therefore

$$
X^{2}+Y^{2}+(Z / 2)^{2}=\left(g_{1}^{0}\right)^{2}+\left(g_{1}^{1}\right)^{2}+\left(h_{1}^{1}\right)^{2}, \quad(Z / 2) \cos \theta+X \sin \theta=-g_{1}^{0},
$$

and

$$
\cos \theta_{p}=-g_{1}^{0} /\left[\left(g_{1}^{0}\right)^{2}+\left(g_{1}^{1}\right)^{2}+\left(h_{1}^{1}\right)^{2}\right]^{1 / 2} .
$$

This is the trivial result that the VGP colatitude $\theta_{p}$ (longitude $\phi_{p}$ also) does not depend on the position of observation $(\theta, \phi)$, when nondipole terms are absent. 


\subsection{Combination of the axial dipole and a nondipole term}

Assume that the magnetic field is made of only two components, one the axial dipole $g_{1}^{0}$ and the other a nondipole component $g_{\ell}^{m}$. We also assume that the dipole has the present (normal) polarity and that its magnitude is much larger than that of the nondipole term, i.e.,

$$
g_{1}^{0}<0, \quad \xi_{\ell}^{m}=g_{\ell}^{m} / g_{1}^{0}, \quad\left|\xi_{\ell}^{m}\right| \ll 1 .
$$

For this case we have from (7)

$$
\begin{aligned}
Z / 2 & =-g_{1}^{0} \cos \theta-\frac{\ell+1}{2} g_{\ell}^{m} P_{\ell}^{m} \cos m \phi, \\
X & =-g_{1}^{0} \sin \theta+g_{\ell}^{m}\left(d P_{\ell}^{m} / d \theta\right) \cos m \phi, \\
Y & =m g_{\ell}^{m} P_{\ell}^{m} \sin m \phi / \sin \theta,
\end{aligned}
$$

and the VGP colatitude is calculated from (9)

$$
\begin{aligned}
\cos \theta_{p}= & \frac{-g_{1}^{0}+g_{\ell}^{m} A_{l m} \cos m \phi}{\left[\left(g_{1}^{0}\right)^{2}-2 g_{1}^{0} g_{\ell}^{m} A_{l m} \cos m \phi+\left(g_{\ell}^{m}\right)^{2}\left(B_{l m l m}^{2} \cos ^{2} m \phi+C_{l m}^{2} \sin ^{2} m \phi\right)\right]^{1 / 2}} \\
= & \left(1-\xi_{\ell}^{m} A_{l m} \cos m \phi\right) \\
& \times\left[1-2 \xi_{\ell}^{m} A_{l m} \cos m \phi+\left(\xi_{\ell}^{m}\right)^{2}\left(B_{l m l m}^{2} \cos ^{2} m \phi+C_{l m}^{2} \sin ^{2} m \phi\right)\right]^{-1 / 2} \\
= & 1-\frac{1}{2}\left(\xi_{\ell}^{m}\right)^{2}\left[\left(B_{l m l m}^{2}-A_{l m}^{2}\right) \cos ^{2} m \phi+C_{l m}^{2} \sin ^{2} m \phi\right]+\ldots
\end{aligned}
$$

where

$$
\begin{gathered}
A_{l m}=\sin \theta \frac{d P_{\ell}^{m}}{d \theta}-\frac{\ell+1}{2} \cos \theta P_{\ell}^{m}, \quad C_{l m}=\frac{m P_{\ell}^{m}}{\sin \theta}, \\
B_{l m l^{\prime} m^{\prime}}^{2}=\frac{d P_{\ell}^{m}}{d \theta} \frac{d P_{\ell^{\prime}}^{m^{\prime}}}{d \theta}+\frac{\ell+1}{2} \frac{\ell^{\prime}+1}{2} P_{\ell}^{m} p_{\ell^{\prime}}^{m^{\prime}} .
\end{gathered}
$$

Because of the assumption that $\left|\xi_{\ell}^{m}\right| \ll 1, \theta_{p}$ is also a small angle and we can use the approximation $\cos \theta_{p}=1-\frac{1}{2} \theta_{p}^{2}+\cdots$, which leads to

$$
\theta_{p}^{2}=\left(\xi_{\ell}^{m}\right)^{2}\left[\left(\cos \theta \frac{d P_{\ell}^{m}}{d \theta}+\frac{\ell+1}{2} \sin \theta P_{\ell}^{m}\right)^{2} \cos ^{2} m \phi+\left(\frac{m P_{\ell}^{m}}{\sin \theta}\right)^{2} \sin ^{2} m \phi\right] .
$$

This gives the squared value of the colatitude of the VGP corresponding to a single measurement of the magnetic field direction taken at one site. If values of $\theta_{p}^{2}$ corresponding to many VGPs are averaged, we obtain the angular variance of the VGPs with respect to the geographic pole.

\subsection{General case}

When all the Gauss coefficients are considered, we have to use the full formula (7) for the field components. The derivation of the colatitude $\theta_{p}$ of the pole proceeds in the same manner as described above. The expression for $\cos \theta_{p}$ for the general case is

$$
\begin{aligned}
\cos \theta_{p}= & \left(1+\sum_{\ell} \sum_{m}^{\prime} A_{l m} \xi_{\ell}^{m} \cos m \phi\right)\left[1-2 \sum_{\ell} \sum_{m}^{\prime} A_{l m}\left(\xi_{\ell}^{m} \cos m \phi+\eta_{\ell}^{m} \sin m \phi\right)\right. \\
& +\sum_{\ell} \sum_{m}^{\prime} \sum_{\ell^{\prime}} \sum_{m^{\prime}}^{\prime}\left\{B_{l m l^{\prime} m^{\prime}}^{2}\left(\xi_{\ell}^{m} \cos m \phi+\eta_{\ell}^{m} \sin m \phi\right)\left(\xi_{\ell^{\prime}}^{m^{\prime}} \cos m^{\prime} \phi+\eta_{\ell^{\prime}}^{m^{\prime}} \sin m^{\prime} \phi\right)\right. \\
& \left.\left.+C_{l m} C_{l^{\prime} m^{\prime}}\left(\xi_{\ell}^{m} \sin m \phi-\eta_{\ell}^{m} \cos m \phi\right)\left(\xi_{\ell^{\prime}}^{m^{\prime}} \sin m^{\prime} \phi-\eta_{\ell^{\prime}}^{m^{\prime}} \cos m^{\prime} \phi\right)\right\}\right]^{-1 / 2}
\end{aligned}
$$




$$
\begin{aligned}
= & 1-\frac{1}{2} \sum_{\ell} \sum_{m} \sum_{\ell^{\prime}} \sum_{m^{\prime}}^{\prime}\left[\left(B_{l m l^{\prime} m^{\prime}}^{2}-A_{l m} A_{l^{\prime} m^{\prime}}\right)\right. \\
& \times\left(\xi_{\ell}^{m} \cos m \phi+\eta_{\ell}^{m} \sin m \phi\right)\left(\xi_{\ell^{\prime}}^{m^{\prime}} \cos m^{\prime} \phi+\eta_{\ell^{\prime}}^{m^{\prime}} \sin m^{\prime} \phi\right) \\
& \left.+C_{l m} C_{l^{\prime} m^{\prime}}\left(\xi_{\ell}^{m} \sin m \phi-\eta_{\ell}^{m} \cos m \phi\right)\left(\xi_{\ell^{\prime}}^{m^{\prime}} \sin m^{\prime} \phi-\eta_{\ell^{\prime}}^{m^{\prime}} \cos m^{\prime} \phi\right)\right]+\ldots
\end{aligned}
$$

where $A_{l m}, B_{l m l^{\prime} m^{\prime}}, C_{l m}$ were defined above, $\eta_{\ell}^{m}=h_{\ell}^{m} / g_{1}^{0}$, and $\sum_{m}{ }^{\prime}$ indicates that the sum is taken excluding the $m=0$ term when $\ell=1$ (the axial dipole term). Finally, we obtain the results as given below.

$$
\begin{aligned}
\theta_{p}^{2}= & \sum_{\ell} \sum_{m} \sum_{\ell^{\prime}} \sum_{m^{\prime}}\left[\left(\cos \theta \frac{d P_{\ell}^{m}}{d \theta}+\frac{\ell+1}{2} \sin \theta P_{\ell}^{m}\right)\left(\cos \theta \frac{d P_{\ell^{\prime}}^{m^{\prime}}}{d \theta}+\frac{\ell^{\prime}+1}{2} \sin \theta p_{\ell^{\prime}}^{m^{\prime}}\right)\right. \\
& \times\left(\xi_{\ell}^{m} \cos m \phi+\eta_{\ell}^{m} \sin m \phi\right)\left(\xi_{\ell^{\prime}}^{m^{\prime}} \cos m^{\prime} \phi+\eta_{\ell^{\prime}}^{m^{\prime}} \sin m^{\prime} \phi\right) \\
+ & \left.\frac{m m^{\prime} P_{\ell}^{m} p_{\ell^{\prime}}^{m^{\prime}}}{\sin ^{2} \theta}\left(\xi_{\ell}^{m} \sin m \phi-\eta_{\ell}^{m} \cos m \phi\right)\left(\xi_{\ell^{\prime}}^{m^{\prime}} \sin m^{\prime} \phi-\eta_{\ell^{\prime}}^{m^{\prime}} \cos m^{\prime} \phi\right)\right] .
\end{aligned}
$$

\section{Angular Standard Deviation}

When the field is produced by an axial dipole and a nondipole term as in Subsection 2.3, the mean-square value of colatitude of the VGPs at a given colatitude $\theta$ can be obtained from (11) by taking average over $\phi$ :

$$
\overline{\theta_{p}^{2}}=\left[\left(\cos \theta \frac{d P_{\ell}^{m}}{d \theta}+\frac{\ell+1}{2} \sin \theta P_{\ell}^{m}\right)^{2}+\left(\frac{m P_{\ell}^{m}}{\sin \theta}\right)^{2}\right] \frac{1+\delta_{m 0}}{2}\left(\xi_{\ell}^{m}\right)^{2},
$$

where $\delta_{i j}$ is the Kronecker delta, and the overbar indicates the azimuthal average. Obviously, the same results will be obtained if we used $h_{\ell}^{m}$ instead of $g_{\ell}^{m}$ for the nondipole component, with $\xi_{\ell}^{m}$ replaced by $\eta_{\ell}^{m}$. Note that we did not assume any special property for the Gauss coefficient $g_{\ell}^{m}$ or $h_{\ell}^{m}$, except that it is small in comparison with $g_{1}^{0}$.

For a general geomagnetic field, the mean-square colatitude of VGPs can be obtained from (12). In taking the longitudinal average of (12), all the cross terms of the form $\xi_{\ell}^{m} \eta_{\ell^{\prime}}^{m^{\prime}}$ vanish because of the orthogonality of $\cos m \phi$ and $\sin m^{\prime} \phi$. The other cross terms $\xi_{\ell}^{m n} \xi_{\ell^{\prime}}^{m n^{\prime}}$ and $\eta_{\ell}^{m} \eta_{\ell^{\prime}}^{m n^{\prime}}$ also disappear if the orders are not the same $\left(m \neq m^{\prime}\right)$. Thus the result of averaging is

$$
\begin{aligned}
\overline{\theta_{p}^{2}}=\sum_{\ell} \sum_{\ell^{\prime}} \sum_{m}\left[\left(\cos \theta \frac{d P_{\ell}^{m}}{d \theta}+\frac{\ell+1}{2} \sin \theta P_{\ell}^{m}\right)\left(\cos \theta \frac{d P_{\ell^{\prime}}^{m}}{d \theta}+\frac{\ell^{\prime}+1}{2} \sin \theta P_{\ell^{\prime}}^{m}\right)\right. \\
\left.+\frac{m^{2} P_{\ell}^{m} P_{\ell^{\prime}}^{m}}{\sin ^{2} \theta}\right] \frac{1+\delta_{m 0}}{2}\left(\xi_{\ell}^{m} \xi_{\ell^{\prime}}^{m}+\eta_{\ell}^{m} \eta_{\ell^{\prime}}^{m}\right) .
\end{aligned}
$$

Again, we did not assume any special property for the Gauss coefficients. The vanishing of the cross terms is a consequence of the orthogonality of trigonometric functions. Note that we now reverted to the unprimed sum for $m$, because the term for the axial dipole in the above expression is actually zero, reflecting the fact that changes in the axial dipole moment do not produce dispersion of VGP directions.

To obtain a paleosecular variation (PSV) model, we must assume how the Gauss coefficients change with time. For this purpose, it may be reasonable to assume that they are uncorrelated random variables. Moreover, it is quite natural to think that $\xi_{\ell}^{m}$ and $\eta_{\ell}^{m}$ have the same variances 
$\sigma_{\ell m}^{2}$, since they represent the same harmonic displaced in the azimuthal direction. With these assumptions, we have

$$
\left\langle\theta_{p}^{2}\right\rangle=\sum_{\ell} \sum_{m}\left[\left(\cos \theta \frac{d P_{\ell}^{m}}{d \theta}+\frac{\ell+1}{2} \sin \theta P_{\ell}^{m}\right)^{2}+\left(\frac{m P_{\ell}^{m}}{\sin \theta}\right)^{2}\right] \sigma_{\ell m}^{2},
$$

where angle brackets indicate the temporal average (as well as azimuthal average) of the value. The square root of $\left\langle\theta_{p}^{2}\right\rangle$ can be regarded as the ASD of the poles for that latitude if $\Delta_{i}$ in (1) is defined as the angular distance from the rotational axis to a VGP. We therefore have obtained an expression describing the dependence of the ASD on latitude for geomagnetic field models in which the Gauss coefficients are regarded as uncorrelated random variables. It is noteworthy that we did not need to assume anything about the mean of these random variables. They do not enter into the expression of VGP dispersion at this level of approximation.

Figure 3 illustrates the change with latitude of the values in the square bracket in Eq. (13) for various low degree harmonics. The curves can be regarded the ASD-latitude relation if the field is produced by axial dipole and only one nondipole harmonic. For the complete model, the variances from various harmonics should be added to calculate the total variance as in (13). For most harmonics the ASD generally increases with latitude, but the manner of increase is different for each harmonic, and some even show the opposite trend (e.g., $g_{2}^{0}, g_{3}^{3}$ ). We will postpone the discussion of this behavior to a later section.

Although (13) is complete in expressing a PSV model, it contains too many parameters if we have to specify each $\sigma_{\ell m}$ separately. In order to reduce the parameters to some reasonable

\section{ASD for Single Gauss Coefficient}
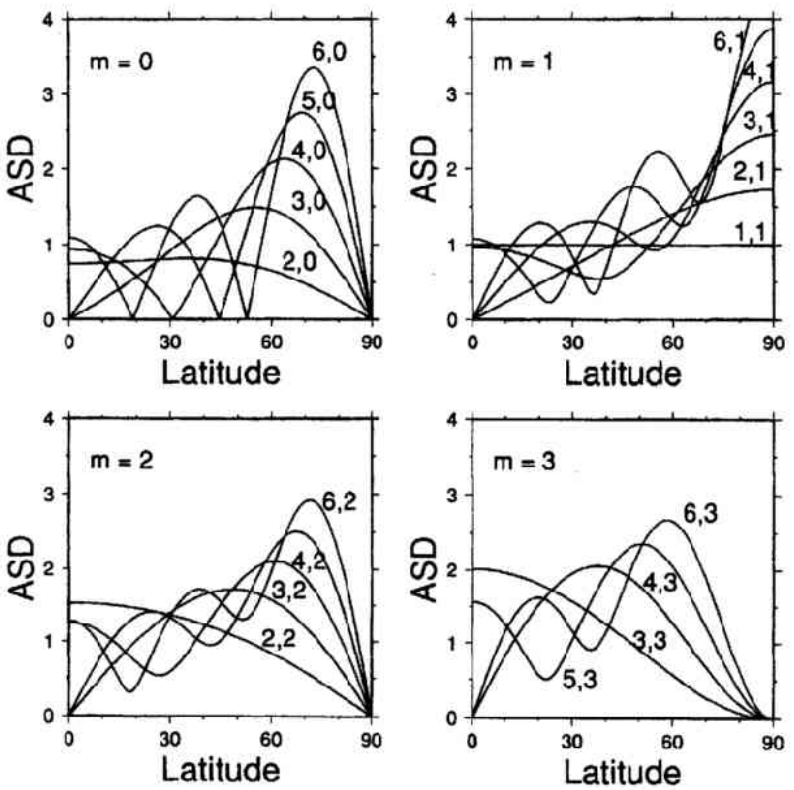

Fig. 3. Angular standard deviation (ASD) of the pole with respect to the rotational axis produced by a magnetic field composed of the axial dipole term and a single nondipole term. The degree and order $(\ell, m)$ of the harmonic is indicated near each curve. The ordinate shows the ASD in units of radian for one standard deviation of $\xi_{\ell}^{m}$ or $\eta_{\ell}^{m}$, while the abscissa gives the latitude in degrees. 
number, we may further assume that the variables of the same order $\ell\left(\xi_{\ell}^{m}\right.$ and $\left.\eta_{\ell}^{m}\right)$ have the same variance $\sigma_{\ell}^{2}$, as was suggested by Constable and Parker (1988), although Harrison and Huang (1990) and Harrison (1994) observed that low-order coefficients tend to have larger magnitudes than high-order coefficients. Then we finally obtain

$$
\left\langle\theta_{p}^{2}\right\rangle=\sum_{\ell} \sigma_{\ell}^{2} \sum_{m}\left[\left(\cos \theta \frac{d P_{\ell}^{m}}{d \theta}+\frac{\ell+1}{2} \sin \theta P_{\ell}^{m}\right)^{2}+\left(\frac{m P_{\ell}^{m}}{\sin \theta}\right)^{2}\right] .
$$

Once the equality of variances for harmonics of the same degree has been assumed, we can further reduce the above expression by using the following relations which can be derived from the addition theorem of Legendre functions (see Constable and Parker, 1988)

$$
\begin{gathered}
\sum_{m}\left[P_{\ell}^{m}(\cos \theta)\right]^{2}=P_{\ell}(1)=1, \quad \sum_{m} \frac{d P_{\ell}^{m}}{d \theta} P_{\ell}^{m}=0, \\
\sum_{m}\left(\frac{d P_{\ell}^{m}}{d \theta}\right)^{2}=\sum_{m}\left(\frac{m P_{\ell}^{m}}{\sin \theta}\right)^{2}=P_{\ell}^{\prime}(1)=\frac{\ell(\ell+1)}{2} .
\end{gathered}
$$

Calculation yields

$$
\begin{aligned}
\left\langle\theta_{p}^{2}\right\rangle & =\sum_{\ell=1}^{\infty}\left[\cos ^{2} \theta \sum_{m}\left(\frac{d P_{\ell}^{m}}{d \theta}\right)^{2}+\frac{(\ell+1)^{2}}{4} \sin ^{2} \theta \sum_{m}\left(P_{\ell}^{m}\right)^{2}+\sum_{m}\left(\frac{m P_{\ell}^{m}}{\sin \theta}\right)^{2}\right] \sigma_{\ell}^{2} \\
& =\sum_{\ell=1}^{\infty}\left[\frac{\ell(\ell+1)}{2}\left(\cos ^{2} \theta+1\right)+\frac{(\ell+1)^{2}}{4} \sin ^{2} \theta\right] \sigma_{\ell}^{2} \\
& =\sum_{\ell=1}^{\infty} \frac{1}{4}(\ell+1)\left[(3 \ell+1)+(\ell-1) \cos ^{2} \theta\right] \sigma_{\ell}^{2} .
\end{aligned}
$$

This gives the squared value of ASD of poles for PSV models in which the Gauss coefficients of the nondipole terms are random variables with zero mean and variance $\sigma_{\ell}^{2}$ which depends only on the degree of the harmonic $\ell$.

If we take for the variance of the nondipole terms the form assumed by Constable and Parker as given in (4), we obtain the angular dispersion of VGP as

$$
\begin{aligned}
\left\langle\theta_{p}^{2}\right\rangle & =2 \sigma_{1}^{2}+\frac{\alpha^{2}}{4} \sum_{\ell=2}^{\infty}\left[\left(\frac{3}{2}-\frac{1}{2} \frac{1}{2 \ell+1}\right)+\left(\frac{1}{2}-\frac{3}{2} \frac{1}{2 \ell+1}\right) \cos ^{2} \theta\right]\left(\frac{c}{a}\right)^{2 \ell} \\
& =2 \sigma_{1}^{2}+\frac{\alpha^{2}}{8}\left[\frac{(c / a)^{4}}{1-(c / a)^{2}}\left(3+\cos ^{2} \theta\right)-\left(\frac{a}{c} \tanh ^{-1} \frac{c}{a}-1-\frac{c^{2}}{3 a^{2}}\right)\left(1+3 \cos ^{2} \theta\right)\right],
\end{aligned}
$$

where the first term on the right hand side indicates the contribution from the equatorial dipole components. The above equation yields the numerical values for the case in which the sources are assumed to lie at the core-mantle boundary $(c / a=0.547)$,

$$
\left\langle\theta_{p}^{2}\right\rangle=2 \sigma_{1}^{2}+0.0450 \alpha^{2}\left(1+0.164 \cos ^{2} \theta\right) .
$$

\section{Evaluation of Paleosecular Variation Models}

With the expressions derived earlier, we can analyze and compare various PSV models in terms of the ASD of the VGPs they predict. Before doing that, we must make one point clear. 
Constable and Parker (1988) assumed that $g_{\ell}^{m}$ and $h_{\ell}^{m}$ are the independent Gaussian statistical variables, while we assumed that the ratios $g_{\ell}^{m} / g_{1}^{0}$ and $h_{\ell}^{m} / g_{1}^{0}$ are the random variables, presumably obeying a normal distribution. The two assumptions are certainly different. However, it can be shown that the difference is quite small and we can freely change from one assumption to the other.

Assume that $g_{1}^{0}$ obeys a normal distribution with mean $x_{0}$ and variance $\sigma_{0}^{2}$, while $g_{\ell}^{m}$ follows a zero-mean normal distribution with variance $\sigma_{\ell}^{2}$. The distribution of $z=g_{\ell}^{m} / g_{1}^{0}$ can then be calculated as

$$
\begin{aligned}
p(z) & =\frac{1}{2 \pi \sigma_{0} \sigma_{\ell}} \int_{-\infty}^{\infty} \exp \left[-\frac{\left(x-x_{0}\right)^{2}}{2 \sigma_{0}^{2}}-\frac{x^{2} z^{2}}{2 \sigma_{\ell}^{2}}\right] x d x \\
& =\frac{x_{0} \sigma_{\ell}^{2}}{\sqrt{2 \pi}\left(\sigma_{0}^{2} z^{2}+\sigma_{\ell}^{2}\right)^{3 / 2}} \exp \left[-\frac{x_{0}^{2} z^{2}}{2\left(\sigma_{0}^{2}+\sigma_{\ell}^{2}\right)}\right] .
\end{aligned}
$$

Since the exponential term quickly decays as the numerator in the square bracket becomes large, it is possible to expand the above expression at small $z$. Putting $q=\left(\sigma_{0} / \sigma_{\ell}\right)^{2}$ and $\sigma=\sigma_{\ell} / x_{0}$, we obtain

$$
\begin{aligned}
p(z) & =\frac{1}{\sqrt{2 \pi} \sigma\left(1+q z^{2}\right)^{3 / 2}} \exp \left[-\frac{z^{2}}{2 \sigma^{2}\left(1+q z^{2}\right)}\right] \\
& =\frac{1}{\sqrt{2 \pi} \sigma}\left(1-\frac{3}{2} q z^{2}+\frac{15}{8} q^{2} z^{4}+\ldots\right)\left[1-\frac{z^{2}}{2 \sigma^{2}}\left(1-q z^{2}+\ldots\right)+\frac{z^{4}}{8 \sigma^{4}}(1-\ldots)-\ldots\right] \\
& =\frac{1}{\sqrt{2 \pi} \sigma}\left[1-\frac{z^{2}}{2 \sigma^{2}}\left(1+3 \sigma^{2} q\right)+\frac{z^{4}}{8 \sigma^{4}}\left(1+10 \sigma^{2} q+15 \sigma^{4} q^{2}\right)+\ldots\right] .
\end{aligned}
$$

Obviously, this expression coincides with the normal distribution with zero mean and variance $\sigma^{2}$, if terms containing $\sigma^{2} q=\left(\sigma_{0} / x_{0}\right)^{2}$ in the last line can be neglected in comparison with 1 . From paleomagnetic observations, the value of $\sigma_{0} / x_{0}$ is estimated to be about $35 \%$ for the last 10 million years interval (Kono, 1971), and is significantly smaller than that for shorter intervals such as the last $10^{4}$ years (e.g., Tanaka et al., 1994). Thus it is permissible to assume that $g_{\ell}^{m} / g_{1}^{0}$ is a Gaussian random variable with zero mean and a variance $\left(\sigma_{\ell} / x_{0}\right)^{2}$.

\subsection{Random source at some depth}

In Section 3, it was shown that, for models in which the Gauss coefficients are treated as random variables of zero mean and variance of the form defined in (4), the angular variance of the poles can be expressed by a very simple latitude dependence of the type

$$
\left\langle\theta_{p}^{2}\right\rangle=2 \sigma_{1}^{2}+\frac{\alpha^{2}}{8}\left[3 f-g+(f-3 g) \sin ^{2} \lambda\right],
$$

where

$$
f=\frac{(c / a)^{4}}{1-(c / a)^{2}}, \quad g=\frac{a}{c} \tanh ^{-1} \frac{c}{a}-1-\frac{1}{3}\left(\frac{c}{a}\right)^{2} .
$$

In the above, $\sigma_{1}$ is the contribution of the equatorial dipole components to the variance and is independent of latitude. In particular, when the source of the magnetic field is assumed to have a white spectrum at the surface of the core, as was assumed by Constable and Parker (1988), the maximum change of the ASD as given by $[(f-3 g) /(3 f-g)]^{1 / 2}$ is only about $8 \%$ when there is no contribution from the equatorial dipole. The range in which the ASD can vary will be even more reduced if the equatorial dipole is included.

From paleomagnetic data, it is well known that the amplitude of secular variation as expressed by the ASD of the VGPs increases monotonically with latitude and the ratio of the ASD at high 
latitudes to that at the equator is about 1.5 (Cox, 1970; McElhinny and Merrill, 1975; see Fig. 1). Thus the change of only $8 \%$ predicted from the PSV model of Constable and Parker is too small and is not consistent with the paleomagnetic observations.

It may be thought possible to enhance the latitudinal change of secular variation, without changing the basic structure of the model, e.g., by changing the depth of the source of the field as given by the ratio $c / a$. When $c / a$ is varied between 0.1 and 0.9 , the value of $(f-3 g) /(3 f-g)$ varies between 0.143 and 0.236 . These, however, correspond to a range of ASD variation of only 6.9 to $11.2 \%$ (see Fig. 4c). Therefore, it is not possible to make the model compatible with paleomagnetic data with a slight change in the source depth. This result holds even if we disregard the contribution from the equatorial dipole terms.

In retrospect, the very weak dependence of the ASD on latitude is a direct consequence of the assumption of the model. By assuming the same variances for rescaled Gauss coefficients $\tilde{g}_{\ell}^{m}$ and $\tilde{h}_{\ell}^{m}$ of different degree and order, we were in effect saying that all the locations at the surface of the earth are statistically indistinguishable. In this respect, the model of Constable and Parker can be regarded as a homogeneously random model. The fact that summation of squared magnetic elements $X^{2}, Y^{2}, Z^{2}$ for $m$ is possible, and the results are independent of $\theta$ as shown in (15), clearly indicates the fact that the statistical properties are independent of position. The weak dependence on latitude is brought out by the inclusion of the axial dipole term which gives a field that is stronger at high latitudes and weaker at low latitudes, and hence is not homogeneous on a spherical surface. To account for a larger latitude dependence of the ASD, it is necessary to assume that some of the harmonics behave differently from others.

Constable and Parker (1988) assumed that, besides the equatorial dipole terms, the axial quadrupole $g_{2}^{0}$ has a nonzero mean, to be consistent with the paleomagnetic observations (Lee, 1983). This choice seems to be appropriate to account for the so-called dipole offset phenomenon deduced from time-averaged paleomagnetic data (Wilson, 1971). However, as for the ASD of the poles, this choice does not improve the situation as they kept the variance of this harmonic the same as others. Increasing its variance produces an adverse result, since the ASD due to $\xi_{2}^{0}$ is nearly constant at low and mid latitudes and decreases at high latitudes (Fig. 2).

From the above analysis, we conclude that models based on Gauss coefficients cannot produce the paleomagnetically observed latitude dependence of PSV if the coefficients are assumed to be random variables of zero mean and variance which depends only on the degree of the harmonic, decreasing with degree in the form of (4).

\subsection{Dipole and quadrupole families}

The above conclusion suggests that the nondipole part of the earth's magnetic field is not completely random in nature and that there must be some structure which results in the observed latitude dependence of the ASD of the poles. One possibility proposed to date is that, because of the inherent symmetry in the dynamo process, there exists some distinction between the harmonics belonging to the dipole family (those with $\ell+m=$ even) and those in the quadrupole family $(\ell+m=$ odd) (McFadden $e t$ al., 1988). From a theoretical point of view, this separation into two families occurs when the velocity field is symmetric and the mean-field generation effect ( $\alpha$-effect) is antisymmetric with respect to the equator (Roberts and Stix, 1972).

Figure 3 shows the effect of a single nondipole harmonic on the ASD of the poles given by (13). As the variance for the general case can be obtained by simply taking the sum of individual variances (under the assumption that Gauss coefficients are uncorrelated random variables), we can construct the ASD curve once the form of $\sigma_{\ell}$ (or $\sigma_{\ell m}$, if similarity of variables of the same degree is not assumed) is given. In considering its form, we should take note of the fact that the power $R_{\ell}$ shows an exponential drop, because such behavior is quite reasonable from the geometrical decay of the magnetic field generated by the dynamo mechanism in the core. Therefore, although the homogeneously random model failed, as shown earlier, we can assume that $\sigma_{\ell}$ of 

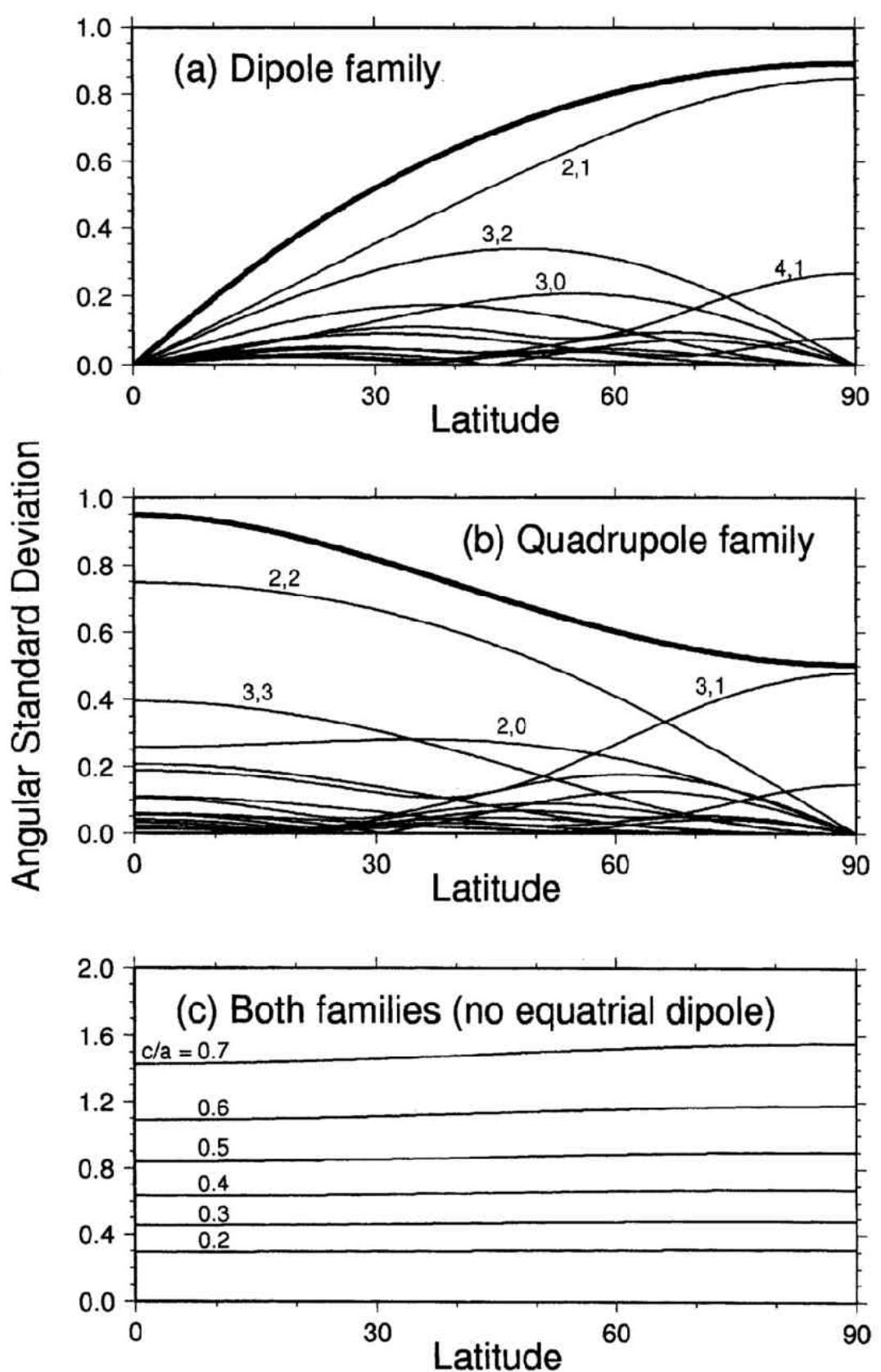

Fig. 4. The ASD of the poles for a magnetic field composed of the axial dipole and nondipole fields of the dipole family (a), and the axial dipole and the quadrupole family (b), and for the model containing all the harmonics (c). The ASD is expressed in the unit of $\sigma_{1}$, which is the ASD caused by the equatorial dipole component $g_{1}^{1}$ or $h_{1}^{1}$. In (a) and (b), thin lines are the contributions from each harmonic with its variance varying as (4), and small numbers indicate the degree and order $(\ell, m)$ of some of the most important harmonics, while the thick line shows the total ASD (summed for $2 \leq \ell \leq 8$ ). In (c), the source depth is changed by changing the values of $c / a$, but the results show very little variation with latitude. 
both the dipole and quadrupole families change as (4) but with different constant terms. This is similar to the assumption used in the model of McFadden et al. (1988). Figure 4 shows the dependence of the ASD on latitude for a magnetic field solely composed of the dipole family (a) or quadrupole family (b). As stated earlier, the combination of the two families with variances obeying (4) yields very little latitude dependence (Fig. 4c), but the dipole family and quadrupole family separately give clear latitude dependences of the ASD.

The dipole family ASD increases monotonically with latitude, reminiscent of the $A \lambda$ term in the model of McFadden et al. (1988), which they ascribed to the combined effect of all the dipole family terms. However, on closer examination, it becomes clear that the shape of the ASD curve is dominated by the low degree harmonics, especially by a quadrupole term $(\ell=2, m=1)$. For the quadrupole family harmonics, the total ASD slowly but significantly decreases with latitude, which is different from what was assumed by McFadden et al. (1988). Obviously this discrepancy is caused by the omission of the equatorial dipole term in Fig. 4, which is constant with latitude and its magnitude of $\sqrt{2}$ is much larger than any other terms or their sum (Fig. 4b). The ASD of the quadrupole family in the absence of the equatorial dipole is also dominated by low degree harmonics; especially by the terms of $\ell=2$ and 3 .

Since the dipole and quadrupole families have different latitude dependent structures as shown in Fig. 4, it is possible to produce various ASD-latitude relations by changing the ratio of the dipole and quadrupole families in the model. Figure 5 shows the ASD obtained by mixing various amounts of dipole and quadrupole family harmonics, each of which have the variances

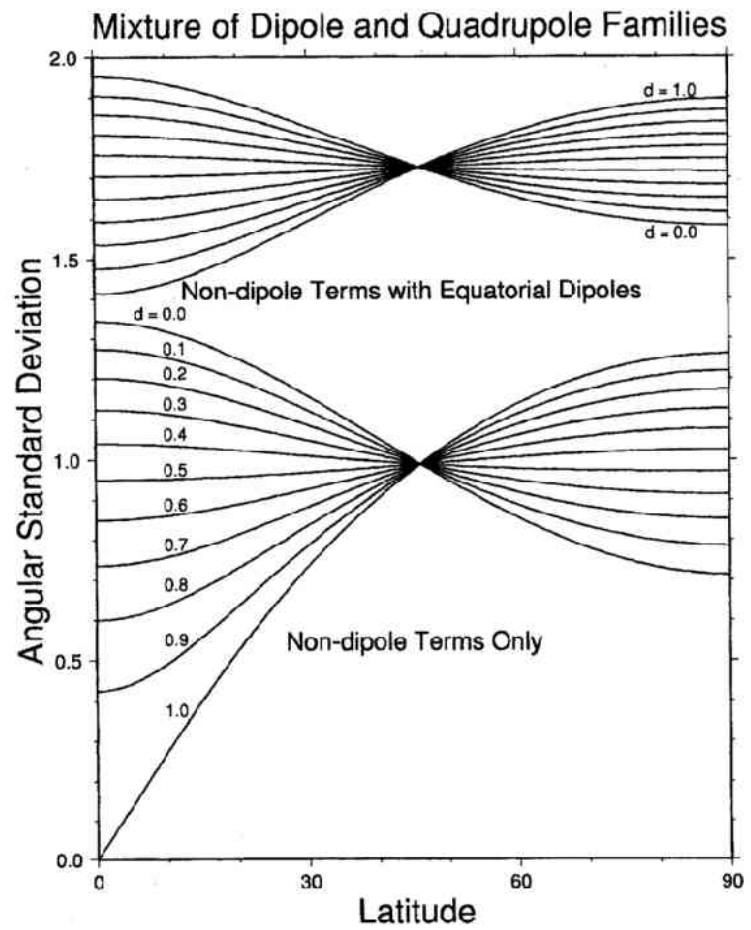

Fig. 5. The ASD of the poles for various mixtures of dipole and quadrupole family harmonics. Cases with and without the equatorial dipole terms $g_{1}^{1}$ and $h_{1}^{1}$ are shown. $d$ indicates the ratio of the dipole family and quadrupole family harmonics as defined in (17). Note that $d=1$ and $d=0$ curves for "non-dipole terms only" correspond to $\sqrt{2}$ times the thick lines in Figs. 4(a) and (b). 
given by (4). In this case, the ASD is given by

$$
\begin{aligned}
\left\langle\theta_{p}^{2}\right\rangle & =2 d \sum_{\ell}^{\ell+m=\text { odd }}\left[\left(\cos \theta \frac{d P_{\ell}^{m}}{d \theta}+\frac{\ell+1}{2} \sin \theta P_{\ell}^{m}\right)^{2}+\left(\frac{m P_{\ell}^{m}}{\sin \theta}\right)^{2}\right] \sigma_{\ell}^{2} \\
& +2(1-d) \sum_{\ell}^{\ell+m=\text { even }}\left[\left(\cos \theta \frac{d P_{\ell}^{m}}{d \theta}+\frac{\ell+1}{2} \sin \theta P_{\ell}^{m}\right)^{2}+\left(\frac{m P_{\ell}^{m}}{\sin \theta}\right)^{2}\right] \sigma_{\ell}^{2},
\end{aligned}
$$

where $d$ is the ratio of dipole family in the model, and $d=1 / 2$ corresponds to the homogeneous case. As can be seen from this figure, different combination of dipole and quadrupole families will yield various latitude dependences. In the homogeneous case, there is very little latitude variation as stated earlier. The amplitude of the ASD decreases drastically if the contribution from the equatorial dipole as defined by (4) is added. In any case, the form of latitude dependence of dipole and quadrupole families as proposed by McFadden et al. (1988) seems to be dominated by the low degree harmonics, especially the dipole terms $g_{1}^{1}$ and $h_{1}^{1}$ in the quadrupole family and the quadrupole term $g_{2}^{1}$ in the dipole family.

\section{Discussion and Conclusions}

We have clarified how the magnetic field represented by a potential which is described in terms of Gauss coefficients is mapped into virtual poles, when the geocentric dipole is assumed to be dominant. As mapping to the VGP is a standard procedure in paleomagnetism, the methods presented in this study enable us to compare paleomagnetic data with the more complete potential representation of the magnetic field.

We have shown in the last section that Constable and Parker's (1988) model, although quite attractive, gives a nearly constant ASD irrespective of latitude. In their model, the Gauss coefficients except the dipole terms and $g_{2}^{0}$ were treated, after renormalization, as samples of the same Gaussian process. Our results show that such an assumption of homogeneous randomness is perhaps too naïve. The geomagnetic field as seen from paleomagnetic data (Fig. 1) has a definite structure which cannot be reproduced by a homogeneous model such as that of Constable and Parker. In a study of the recent geomagnetic field, Harrison and Huang (1990) and Harrison (1994) noted that lower order harmonics tend to be larger than the higher order harmonics of the same degree $\ell$. This shows that the assumption of Constable and Parker is not consistent with the recent field.

One way to include the latitude dependent structure in a similar model is to divide the field into the dipole and quadrupole families as suggested by McFadden et al. (1988). This method actually yields various latitude dependences of the ASD of the poles when the ratio of the dipole family to the total magnetic field is changed. However, on closer examination, it was found that the manner of latitude variation of the two families or their combination is strongly controlled by the low degree harmonics, especially by the terms with $\ell=1$ and 2 . This is because of the geometrical decay of the harmonics as shown by (4). Thus the postulated variance of the model of McFadden et al. given in (5), $A^{2} \lambda^{2}+B^{2}$, can essentially be produced by only the two terms $g_{1}^{1}\left(h_{1}^{1}\right)$ and $g_{2}^{1}\left(h_{2}^{1}\right)$. Although the dipole/quadrupole family model can be made consistent with the observed paleomagnetic data if the variances of these two terms are given appropriate magnitudes, it does not mean that such a model is preferred from the data. In fact, because of the small power contained in the higher modes at the surface, the terms with degree 3 and higher do not change the latitude dependence of the ASD in any significant way. In other words, the data do not have the ability to discriminate the higher degree harmonics.

Consideration of dynamo mechanism does not support the separation of the two families either. It is well known that all the $\alpha^{2}$ - and $\alpha \omega$-dynamos studied so far give very similar critical 
Reynolds numbers for the solutions of the two families (Roberts, 1972; Kono and Roberts, 1991). This means that the magnetic fields of the two families can be excited with similar ease. This tendency continues even when the system is in a slightly nonlinear regime (Kono and Roberts, 1992). Separation of the two families results if the velocity field is symmetric and the $\alpha$-effect is antisymmetric with respect to the equator (Roberts and Stix, 1972). However, such a condition may not prevail in the earth's core. In fact, inversion of the recent secular variation yields a velocity field at the surface of the core which is significantly different from the required symmetry, and there are even flows crossing the equator (e.g., Bloxham et al., 1989).

When Cox (1970) proposed classical PSV models, he noted the latitude independent nature of the ASD due to the dipole term but did not specify how the observed latitude dependence $F(\lambda)$ of the nondipole terms can be achieved. Later, McFadden et al. (1988) showed from the analysis of the present magnetic field that dipole and quadrupole family ASDs are proportional to and independent of latitude. Because the ASD-latitude relation is quite similar for the present field and for the last 5 million years, they concluded that the dipole/quadrupole family decoupling is a fundamental feature of the geodynamo. The present study, however, suggests that this conclusion may be premature. In fact, there is no resolving power for the higher harmonics in paleomagnetic data as given by the ASD-latitude relation. All we can say is that some of the harmonics of the form $g_{\ell}^{1}\left(h_{\ell}^{1}\right)$ must have a large amplitude compared with the other terms, which may obey the ordinary power law of the form (4). This situation is necessary to produce an ASD which increases with latitude (Figs. 3 and 4).

Over all, our conclusion is similar to that of Cox, with slight modification. The ASD of the poles can be divided into two parts; one due to the dipole wobble and the other due to nondipole terms $g_{2}^{1}\left(h_{2}^{1}\right)$ and, to a lesser extent, $g_{3}^{1}\left(h_{3}^{1}\right)$. There are other harmonics which show a tendency to increase the ASD with latitude (Fig. 3), but their contribution to the total ASD is negligible because of the geometrical decay of the higher harmonics (Fig. 4). Thus, the most important contributions to the ASD come from the low-degree Gauss coefficients, probably $g_{1}^{1}\left(h_{1}^{1}\right), g_{2}^{0}$, and $g_{2}^{1}\left(h_{2}^{1}\right)$. We propose that there is some mechanism in the geodynamo which keeps not only the $g_{2}^{0}$ but the $g_{2}^{1}\left(h_{2}^{1}\right)$ harmonics persistently larger than other non-dipole terms. Although we do not present a definite PSV model, it can be constructed easily by combining only these low degree harmonics with appropriate weights.

In the past, the importance of the $g_{2}^{0}$ term was emphasized because of the observation of the dipole offset (Wilson, 1971), and the study of secular variation showed that the terms which are mostly responsible for the westward drift are the sectorial harmonics $g_{\ell}^{\ell}\left(h_{\ell}^{\ell}\right)$ (Yukutake and Tachinaka, 1968). However, the importance of low-degree $g_{\ell}^{1}\left(h_{\ell}^{1}\right)$ harmonics has not been well recognized, although there have been some attempts to resolve the non-axisymmetric terms in the secular variation (e.g., Merrill and McElhinny, 1977). One exception is the work of Tsunakawa (1988), who concluded that in the non-dipole terms $h_{2}^{1}$ is dominant, which agrees quite well with our results.

Tsunakawa (1988) compiled worldwide PSV data from lavas of Brunhes chron and noticed that the VGP distribution is not azimuthally isotropic and that the shape of the distribution depends on the longitude of the observation site. He proposed that the elongation of the VGP distribution is caused by a non-dipole term which varies in proportion to $g_{1}^{0}$ while the dipole direction changes randomly. He chose seven Gauss coefficients which showed a high correlation with $g_{1}^{0}$ over the past 200 years, and assumed that these Gauss coefficients are given by linear relations with $g_{1}^{0}$. Tsunakawa concluded that there is a large amount of the $h_{2}^{1}$ component in the recent geomagnetic field to explain the elongated distribution of VGPs. In our study axial symmetry was assumed so that dependence on longitude is not taken into account. It may be worthwhile to investigate possible azimuthal non-uniformity as suggested by Tsunakawa (1988). We must note, however, that his conclusion was obtained by looking at Gauss coefficients which are correlated with the temporal change of $g_{1}^{0}$ rather than treating them as uncorrelated 
random variables.

We are very grateful to Andrew Jackson who kindly supplied the Gauss coefficients as well as the retrieval program for the Bloxham and Jackson models. We thank Masaki Higurashi who checked the validity of some of the formulas in this work, Chris Harrison and Cathy Constable for helpful comments on the manuscript, and Gillian Turner for polishing up the English expression. The figures in this paper were produced by the GMT free software package developed by Wessel and Smith (1991).

\section{REFERENCES}

Bloxham, J. and A. Jackson, Time-dependent mapping of the magnetic field at the core-mantle boundary, $J$. Geophys. Res., 97, 19537-19563, 1992.

Bloxham, J., D. Gubbins, and A. Jackson, Geomagnetic secular variation, Phil. Trans. R. Soc. Lond., A329, 415-502, 1989.

Constable, C. G. and R. L. Parker, Statistics of the geomagnetic secular variation for the past 5 m.y., J. Geophys. Res., 93, 11569-11581, 1988.

Cox, A., Latitude dependence of the angular dispersion of the geomagnetic field, Geophys. J. R. Astron. Soc., 20, $253-269,1970$.

Fisher, R., Dispersion on a sphere, Proc. R. Soc. Lond., A217, 295-305, 1953.

Harrison, C. G. A., An alternative picture of the geomagnetic field, J. Geomag. Geoelectr., 46, 127-142, 1994.

Harrison, C. G. A. and Q. Huang, Rates of change of the earth's magnetic field measured by recent analysis, $J$. Geomag. Geoelectr., 42, 897-928, 1990.

Kono, M., Intensity of the earth's magnetic field in Pliocene and Pleistocene in relation to the amplitude of mid-ocean ridge magnetic anomalies, Earth Planet. Sci. Lett., 11, 10-17, 1971.

Kono, M. and P. H. Roberts, Small amplitude solutions of the dynamo problem, 1. The adjoint system and its solutions, J. Geomag. Geoelectr., 43, 839-862, 1991.

Kono, M. and P. H. Roberts, Small amplitude solutions of the dynamo problem, 2. The case of $\alpha^{2}$-dynamos, Geophys. Astrophys. Fluid Dynam., 67, 65-85, 1992.

Langel, R. A. and R. H. Estes, A geomagnetic field spectrum, Geophys. Res. Lett., 9, 250-253, 1982.

Lee, S., A study of the time-averaged paleomagnetic field for the past 195 million years, $\mathrm{Ph} . \mathrm{D}$. Thesis, Australian National University, Canberra, 1983.

Lowes, F. J., Spatial power spectrum of the main geomagnetic field, and extrapolation to the core, Geophys. J. R. Astr. Soc., 36, 717-730, 1974.

McElhinny, M. W. and R. T. Merrill, Geomagnetic secular variation over the past 5 m.y., Rev. Geophys. Space Phys., 13, 687-708, 1975.

McFadden, P. L., R. T. Merrill, and M. W. McElhinny, Dipole/quadrupole family modeling of paleosecular variation, J. Geophys. Res., 93, 11583-11588, 1988.

Merrill, R. T. and M. W. McElhinny, Anomalies in the time-averaged paleomagnetic field and their implications for the lower mantle, Rev. Geophys. Space Phys., 15, 309-323, 1977.

Roberts, P. H., Kinematic dynamo models, Phil. Trans. R. Soc. Lond., A272, 663-698, 1972.

Roberts, P. H. and M. Stix, $\alpha$-effect dynamos, by the Bullard-Gellman formalism, Astron. Astrophys., 18, 453-466, 1972.

Tanaka, H., A. Otsuka, T. Tachibana, and M. Kono, Paleointensities for 10-22 ka from volcanic rocks in Japan and New Zealand. Earth Planet. Sci. Lett., 122, 29-42, 1994.

Tsunakawa, H., Geomagnetic secular variation during the Brunhes epoch inferred from the paleomagnetism and the last 200 years geomagnetic field, J. Geomag. Geoelectr., 40, 1365-1385, 1988.

Wessel, P. and W. H. F. Smith, Free software helps map and display data, EOS, Trans. AGU, 72, 441-446, 1991.

Wilson, R. L., Dipole offset-The time average palaeomagnetic field over the past 25 million years, Geophys. $J$ R. Astron. Soc., 22, 491-504, 1971.

Yukutake, T. and H. Tachinaka, The westward drift of the geomagnetic secular variation, Bull. Earthq. Res. Inst., 46, 1075-1102, 1968. 